\title{
Clinical Features and Outcomes of Endoscopic Treatment for Stones in Stemware-Shaped Common Bile Ducts: A Multicenter Data Analysis
}

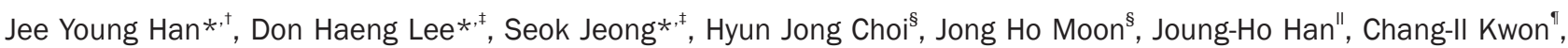 \\ Tae Hoon Lee ${ }^{\#}$, Jong Jin Hyun**, Jae Chul Hwang ${ }^{\dagger \dagger}$, and Dong Hee Koh ${ }^{\ddagger \ddagger}$ \\ *Department of Internal Medicine, Inha University Hospital, Incheon, ${ }^{\dagger}$ Korea Association of Health Promotion, Jeonbuk Branch, Jeonju, \\ ${ }^{\ddagger}$ National Center of Efficacy Evaluation for the Development of Health Products Targeting Digestive Disorders (NCEED), and Utah-Inha \\ DDS \& Advanced Therapeutics Research Center, Incheon, ${ }^{s}$ Digestive Disease Center and Research Institute, Soonchunhyang University \\ Bucheon Hospital, Bucheon, "Division of Gastroenterology, Department of Internal Medicine, Chungbuk National University College of \\ Medicine, Cheongju, "Digestive Disease Center, CHA Bundang Medical Center, CHA University, Seongnam, "Department of Internal Medicine, \\ Soonchunhyang University Cheonan Hospital, Soonchunhyang University School of Medicine, Cheonan, "*Division of Gastroenterology, \\ Department of Internal Medicine, Korea University Ansan Hospital, Korea University School of Medicine, Ansan, ${ }^{\text {tt} D e p a r t m e n t ~ o f ~}$ \\ Gastroenterology, Ajou University School of Medicine, Suwon, and ${ }^{\sharp 1}$ Division of Gastroenterology, Department of Internal Medicine, Dongtan \\ Sacred Heart Hospital, Hwaseong, Korea
}

Background/Aims: Various anatomical features of the biliary tree affect ability to remove difficult common bile duct (CBD) stones. In this study, we evaluated the clinical characteristics and outcomes of the endoscopic treatment of stones in stemware-shaped CBDs. Methods: Thirty-four patients with a stone and a stemware-shaped CBD who were treated at different tertiary referral centers from January 2008 to December 2012 were studied retrospectively. When stone removal failed, percutaneous or direct peroral cholangioscopic lithotripsy, endoscopic retrograde biliary drainage, or surgery was performed as a second-line procedure. Results: The overall success rate of the first-line procedure was $41.2 \%$. Five of the 34 patients $(14.7 \%)$ experienced procedure-related complications. No procedure-related mortality occurred. Mechanical lithotripsy was required to completely remove stones in 13 patients (38.2\%). Conversion to a second-line procedure was required in 20 patients (58.8\%). Mechanical lithotripsy was needed in $75 \%$ and $66.7 \%$ of those with a stone size of $<1 \mathrm{~cm}$ or $\geq 1 \mathrm{~cm}$, respectively. Stone recurrence occurred in two patients (9.1\%) after 6 months and 27 months, respectively. Conclusions: The endoscopic treatment of stones in a stemware-shaped CBD is challenging. The careful assessment of difficult CBD stones is required before endoscopic procedures. (Gut Liver 2015;9:800-804)

Key Words: Common bile duct; Choledocholithiasis; Me- chanical lithotripsy; Cholangiopancreatography, endoscopic retrograde

\section{INTRODUCTION}

Endoscopic retrograde cholangiopancreatography (ERCP) has been substitute for surgery to treat most common bile duct (CBD) stones since it was introduced four decades ago. ${ }^{1}$ About 85\% to $95 \%$ of CBD stones can be successfully removed by conventional ERCP with endoscopic sphincterotomy (EST). ${ }^{2-4}$ However, difficult-to-remove stones need stone-fragmentation methods, such as, mechanical lithotripsy (ML), intraductal shock wave lithotripsy, extracorporeal shock wave lithotripsy, or biliary stenting with chemical dissolution.

Multiple factors, such as, stone number ( $>10)$, size ( $>15 \mathrm{~mm}$ ), shape (barrel-shaped), and location (intrahepatic, cystic duct) can cause difficulties during stone removal..$^{5}$ Recently, Kim et al. ${ }^{6}$ identified anatomical factors of the bile duct that contribute to technical difficulties during the endoscopic clearance of CBD stones in patients with acute distal CBD angulation $\left(\leq 135^{\circ}\right)$ and a short distal CBD arm $(\leq 36 \mathrm{~mm})$. However, no report has associated a stemware-shape and the difficulty of stone removal. In our clinical experience, even a stone diameter of $<1 \mathrm{~cm}$ in a stemware-shaped CBD can cause post-ERCP complications, such as, basket impaction or bile duct perforation during ERCP.

Accordingly, in the current study, we evaluated the clinical characteristics and outcomes of the endoscopic treatment of

Correspondence to: Seok Jeong

Digestive Disease Center, Department of Internal Medicine, Inha University Hospital, 27 Inhang-ro, Jung-gu, Incheon 22332, Korea

Tel: +82-32-890-2548, Fax: +82-32-890-2549, E-mail: inos@inha.ac.kr

Received on October 31, 2014. Revised on December 17, 2014. Accepted on December 26, 2014. Published online June 19, 2015

pISSN 1976-2283 eISSN 2005-1212 http://dx.doi.org/10.5009/gnl14433

Jee Young Han and Don Haeng Lee contributed equally to this work and share first authorship.

@) This is an Open Access article distributed under the terms of the Creative Commons Attribution Non-Commercial License (http://creativecommons.org/licenses/by-nc/4.0) which permits unrestricted non-commercial use, distribution, and reproduction in any medium, provided the original work is properly cited. 
stones in stemware-shaped CBDs.

\section{MATERIALS AND METHODS}

Between January 2008 and December 2012, 34 CBD stone patients who visited eight tertiary referral centers in Korea were enrolled in this study. ERCP images were searched for stemware-shaped CBDs, which were defined as CBDs that met one or more of the following criteria: (1) an intrapancreatic extrahepatic duct (EHD) diameter of $<1 / 2$ of the maximum diameter of extrapancreatic portion of the EHD; (2) an intrapancreatic narrow segment of relatively constant diameter with a difference between the smallest and largest portion of $<5 \mathrm{~mm}$, and must meet the following criteria at the same time; (3) a proximal biliary tree dilation degree, including the intrahepatic duct (IHD), proportional to that of the extrapancreatic CBD (Fig. 1).

Medical records and imaging studies, including abdominal computed tomography, magnetic resonance cholangiopancreatography (MRCP), and ERCP, were retrospectively reviewed. The study protocol was approved by the Institutional Review Board of all participating centers (IUH-IRB 14-058).

\section{Interventions}

Initially, conventional ERCP using an extraction basket and/ or balloon with EST or endoscopic papillary balloon dilation (EPBD) was performed as a first-line procedure in all 34 patients. Multiple procedural sessions were needed to achieve complete CBD clearance in some patients. ML was performed to extract stones in patients with difficult stones. When stone removal failed, a second-line procedure, such as, percutaneous transhepatic cholangioscopic lithotripsy (PTCS-L), endoscopic retrograde biliary drainage (ERBD) with or without oral dissolution treatment (ODT), direct peroral cholangioscopy (POC), or a surgical option was adopted.

\section{Measurement of outcomes}

The primary study endpoint was the overall success rate of complete stone clearance during the first-line procedure. Secondary endpoints included initial procedural success rate for first-line procedures, numbers of first-line procedure sessions required to achieve complete stone clearance, the use of ML, and the overall complication rate (e.g., post-ERCP pancreatitis, cholangitis, cholecystitis, bleeding, perforation, and basket impaction), and rates of conversions to other treatment options such as PTCS-L, surgery, ERBD with ODT, and direct POC. We also evaluated stone recurrence in patients followed up for at least 6 months after stone removal.

\section{RESULTS}

Thirty-four patients with stones in a stemware-shaped CBD were retrospectively enrolled. Baseline characteristics of the study subjects and the characteristics of stones are summarized in Table 1. Median patient age was 56.8 years (range, 26 to 91 years), median total bilirubin was $5.9 \mathrm{mg} / \mathrm{dL}$ (range, 0.4 to 22.3 $\mathrm{mg} / \mathrm{dL}$ ), and the median number of CBD stones per patient was
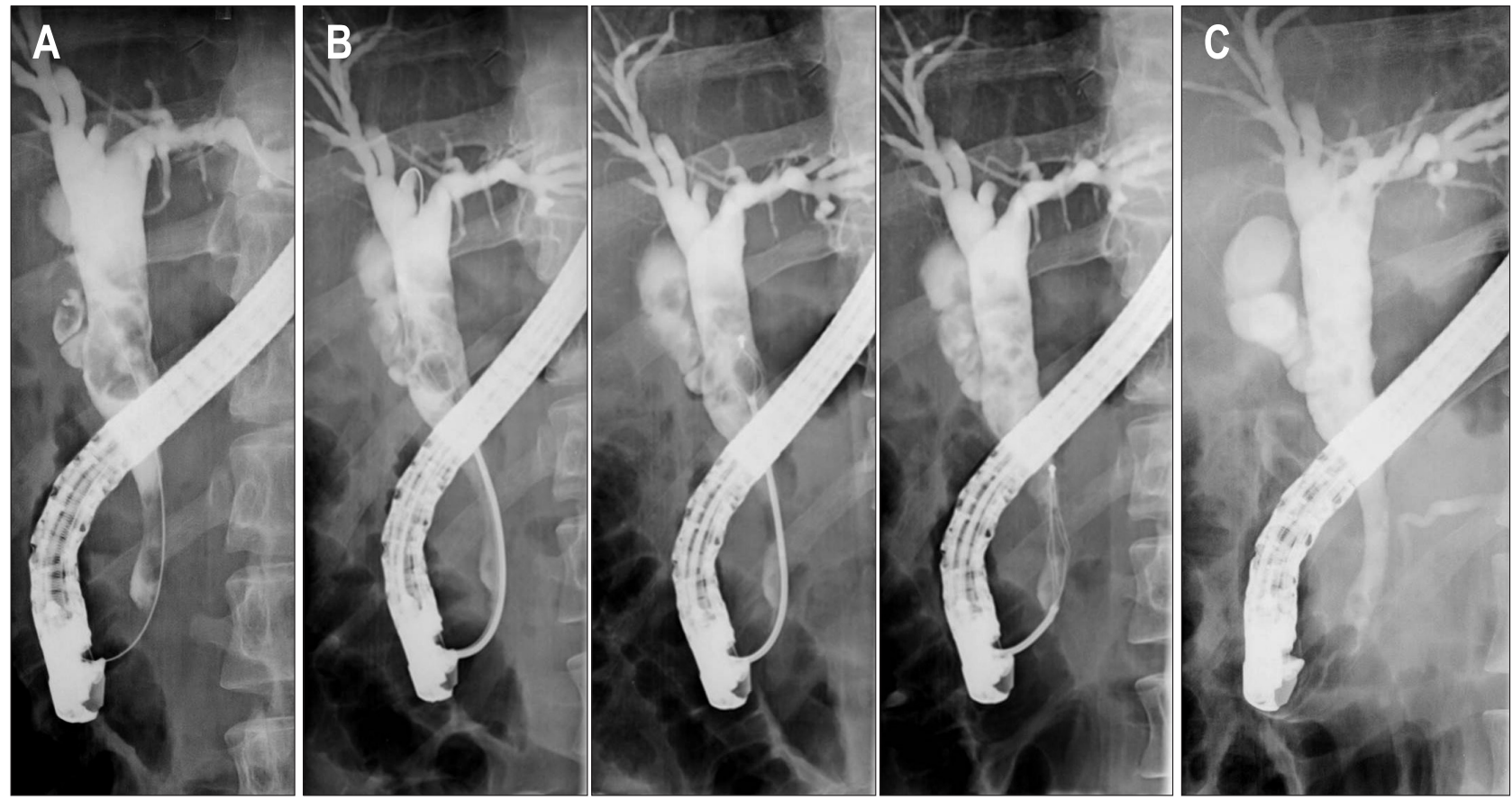

Fig. 1. (A) Endoscopic retrograde cholangiogram showing multiple variably sized stones in a stemware-shaped common bile duct (CBD). (B) Multiple sessions of mechanical lithotripsy were performed in the dilated proximal CBD. (C) No residual stone was observed in the stemware-shaped CBD by cholangiography that was performed after stone removal using a basket and an extraction balloon catheter. 
1 (range, 1 to 10). Median diameter of the extrapancreatic CBD portion was $15.4 \mathrm{~mm}$ (range, 8.5 to $32.1 \mathrm{~mm}$ ), median diameter of the intrapancreatic CBD portion was $5.4 \mathrm{~mm}$ (range, 2.6 to $13.4 \mathrm{~mm}$ ), and the median transverse diameter of stones was $12.3 \mathrm{~mm}$ (range, 1.8 to $30 \mathrm{~mm}$ ).

A post-ERCP complication occurred in five patients (14.7\%); perforation in two, and post-ERCP pancreatitis, basket impaction, and hyperamylasemia in one patient apiece. No mortality occurred (Table 2).

Table 1. Baseline Characteristics of the Patients

\begin{tabular}{lc}
\multicolumn{1}{c}{ Characteristic } & Value \\
\hline No. of patients & 34 \\
Age, yr & $56.8(26-91)$ \\
Sex, male/female & $22 / 12$ \\
No. of CBD stones & $1(1-10)$ \\
Total bilirubin, mg/dL & $5.9(0.4-22.3)$ \\
Diameter, mm & \\
$\quad$ Extrapancreatic portion of CBD & $15.4(8.5-32.1)$ \\
$\quad$ Intrapancreatic portion of CBD & $5.4(2.6-13.4)$ \\
$\quad$ Transverse diameter of stone & $12.3(1.8-30)$ \\
Stone characteristics (brown/black/cholesterol) & $17 / 12 / 5$ \\
\hline
\end{tabular}

Data are presented as number or median (range).

$\mathrm{CBD}$, common bile duct.

Table 2. Safety of Conventional Therapeutic Endoscopic Retrograde Cholangiopancreatography for Stones in Stemware-Shaped Common Bile Ducts

\begin{tabular}{ll}
\hline \multicolumn{1}{c}{ Safety parameter } & Value \\
\hline No. of patients & 34 \\
Complication & $5(14.7)$ \\
Pancreatitis & $1(2.9)$ \\
Cholangitis & 0 \\
Cholecystitis & 0 \\
Perforation & $2(5.9)$ \\
Basket impaction & $1(2.9)$ \\
Hyperamylasemia & $1(2.9)$ \\
Mortality & 0
\end{tabular}

Data are presented as number (\%).
Initial procedural success and overall success rates were 5.9\% and $41.2 \%$, respectively, for first-line procedures, demonstrating the poor efficacy of conventional ERCP for the treatment of stones in a stemware-shaped CBD. Twenty of the 34 patients $(58.8 \%)$ were converted to a second-line procedure (Table 3). One patient who failed the first-line procedure underwent choledocholithotomy as a second-line of procedure and succumbed to acute renal failure 8 days after surgery.

Eight patients had a maximum stone size of $<1 \mathrm{~cm}$ and 26 had one of $>1 \mathrm{~cm}$. Overall success rates for first-line procedures in these two groups were $62.5 \%$ and $34.6 \%$, respectively. ML was needed in 60\% and 66.7\% of patients, respectively, in each group in whom stone retrieval by conventional ERCP succeeded, and $37.5 \%$ and $65.4 \%$, respectively, were converted to a secondline procedure (Table 4). Twenty-two patients were followed up for at least 6 months after stone removal, and only two (9.1\%)

Table 3. Efficacy of Conventional Therapeutic Endoscopic Retrograde Cholangiopancreatography for Stemware-Shaped Common Bile Duct Stones

\begin{tabular}{lc}
\hline \multicolumn{1}{c}{ Efficacy parameter } & Value \\
\hline No. of patients & 34 \\
Success rate & \\
$\quad$ ISR $^{*}$ after first-line procedure & $2(5.9)$ \\
OSR after first-line procedure & $14(41.2)$ \\
Session no. of first-line procedure required & $3 / 10 / 1$ \\
for complete stone removal, 1/2/3 & \\
Total procedure time of successful first-line & $34.5(16.6-72)$ \\
procedure, min & \\
Second-line of procedure & $20(58.8)$ \\
$\quad$ PTCS-L & $2(5.9)$ \\
Surgery & $7(20.6)$ \\
ERBD with/without ODT & $10(29.4)$ \\
Direct peroral cholangioscopy & $3(8.8)$ \\
Stone recurrence rate &
\end{tabular}

Data are presented as number (\%) or median (range).

ISR, initial success rate; OSR, overall success rate; PTCS-L, percutaneous transhepatic cholangioscopic lithotripsy; ERBD, endoscopic retrograde biliary drainage; ODT, oral dissolution treatment.

${ }^{*}$ Complete stone removal during the first ERCP session; ${ }^{\dagger}$ In patients who were followed for at least 6 months after stone removal.

Table 4. Comparison of Outcomes according to the Maximum Stone Size

\begin{tabular}{lcrr}
\hline \multicolumn{1}{c}{ Clinical outcome } & Stone size $<1 \mathrm{~cm}$ & Stone size $\geq 1 \mathrm{~cm}$ & Overall \\
\hline Overall success after first-line procedure & $5 / 8(62.5)$ & $9 / 26(34.6)$ & $14 / 34(41.2)$ \\
Need of mechanical lithotripsy* & $3 / 5(60.0)$ & $6 / 9(66.7)$ & $9 / 14(64.3)$ \\
Conversion to second-line procedure & $3 / 8(37.5)$ & $17 / 26(65.4)$ & $20 / 34(58.8)$ \\
Overall complications & $0 / 8$ & $4 / 26(15.4)$ & $4 / 34(11.8)$ \\
\hline
\end{tabular}

Data are presented as number $(\%)$.

*Use of mechanical lithotripsy in cases of success after the first-line procedure. 
developed stone recurrence. Median follow-up was 24 months (range, 1 to 64 months).

\section{DISCUSSION}

ERCP is one of the most widespread endoscopic methods, and is used to remove CBD stones in most cholelithiasis patients. CBD stones can usually be retrieved endoscopically by conventional ERCP. However, multiple factors can affect the success or failure of endoscopic removal. The reasons why treatment fail are as follows; difficulties accessing the bile duct (periampullary diverticulum, sigmoid-shaped CBD, and altered anatomy due to gastric surgery), a large stone (diameter $>15 \mathrm{~mm}$ ), a large number of stones $(\geq 10)$, an unusual stone shape (barrel-shaped), or stone location (intrahepatic, cystic duct, proximal to a stricture). $^{7}$

Some studies have been conducted on relations between anatomical CBD condition and CBD stone recurrence or the difficulty of endoscopic stone retrieval. Keizman et al. ${ }^{8}$ demonstrated that more acute angulation of the CBD $\left(\leq 145^{\circ}\right)$ influences symptomatic stone recurrence after successful endoscopic treatment. Recently, Kim et al. ${ }^{6}$ first described anatomical bile duct features that increase the technical difficulty of endoscopic stone removal in patients with acute distal CBD angulation and a short distal CBD arm. However, no previous study has addressed the effect of a stemware-shaped CBD on difficult CBD stone removal. In this study, we devised a definition for stemware-shaped CBD and excluded other anatomical anomalies, such as, choledochal cyst and biliary stricture.

In practice, we have experienced many technical difficulties removing stones from a stemware-shaped CBD by ERCP. Even when stones are small, ERCP is laborious, often requires mechanical lithotripsy, and sometimes fails. Furthermore, in such cases, adverse events, such as, basket impaction or perforation, occur frequently. Initially, we considered that failures are caused by a narrow, long, and rigid intrapancreatic portion. Post-ERCP perforation occurred in two of our patients (5.9\%); one during biliary insertion of guidewire and the other after balloon dilation of the papilla. We chose a large diameter balloon catheter for EPBD because extrapancreatic CBD portions were relatively wide and because we failed to recognize stemware-shaped anatomies before and during procedures. Accordingly, this study demonstrates the importance of assessing distal CBD shape by imaging study before the endoscopic procedure, including balloon dilatation, to prevent those avoidable complications.

In the present study, overall success rates of complete bile duct clearance at initial and after first-line procedures were only $5.9 \%$ and $41.2 \%$, respectively. In addition, more than $70 \%$ of patients that underwent successful complete stone removal at first-line procedure needed more than two sessions of ERCP, and for stones of $<1 \mathrm{~cm}$, the overall success rate of first-line procedures was only $62.5 \%$ and ML was needed in $60 \%$ of pa- tients.

The recurrence rate of $\mathrm{CBD}$ stones has been reported to range from $4 \%$ to $24 \% .{ }^{9}$ Recurrence is defined as the development of a stone at least 6 months after complete stone clearance. ${ }^{9}$ The risk factors for recurrence are a bile duct diameter of $>13 \mathrm{~mm}$, periampullary diverticulum (type I or II), CBD angulation, previous history of cholecystectomy, and an age exceeding 80 years. ${ }^{8-11}$ Stemware-shaped CBDs exhibit proximal dilatation and distal stenosis, which could cause bile stasis, and thus, increase the risk of stone recurrence. However, in the current study, the recurrence rate was not too high. Furthermore, comparing our findings with those of previous studies, it would appear stone recurrence is not higher in patients with a stemware-shaped $\mathrm{CBD}$, but the number of patients recruited was too small to determine the real recurrence rate accurately.

Although the present study is limited by its small sample size and retrospective nature, it was conducted on a multicenter basis and describes for the first time the clinical features and outcomes of stones located in stemware-shaped CBDs.

The endoscopic treatment of stones in stemware-shaped CBDs is challenging and requires careful assessment before endoscopic procedure. Endoscopists may need to prepare for mechanical lithotripsy or other treatment options before commencing ERCP even for small stones in stemware-shaped CBDs.

\section{CONFLICTS OF INTEREST}

No potential conflict of interest relevant to this article was reported.

\section{ACKNOWLEDGEMENTS}

The current study was supported by an Inha University Research Grant.

Author's contributions:

Jee Young Han and Don Haeng Lee: conception and design, collection and assembly of data, analysis and interpretation of the data, drafting of the article, provision of study materials, administrative and technical or logistic support.

Seok Jeong: conception and design, provision of study materials, critical revision of the article for important intellectual content, and final approval of the article.

Hyun Jong Choi, Jong Ho Moon, Joung-Ho Han, ChangIl Kwon, Tae Hoon Lee, Jong Jin Hyun, Jae Chul Hwang, and Dong Hee Koh: collection and assembly of the data and provision of study materials and patients

\section{REFERENCES}

1. Binmoeller KF, Schafer TW. Endoscopic management of bile duct stones. J Clin Gastroenterol 2001;32:106-118.

2. Neuhaus H. Endoscopic and percutaneous treatment of difficult 
804 Gut and Liver, Vol. 9, No. 6, November 2015

bile duct stones. Endoscopy 2003;35:S31-S34.

3. Samardzic J, Latic F, Kraljik D, et al. Treatment of common bile duct stones: is the role of ERCP changed in era of minimally invasive surgery? Med Arh 2010;64:187-188.

4. Stromberg C, Nilsson M. Nationwide study of the treatment of common bile duct stones in Sweden between 1965 and 2009. Br J Surg 2011;98:1766-1774.

5. Binmoeller KF, Schafer TW. Endoscopic management of bile duct stones. J Clin Gastroenterol 2001;32:106-118.

6. Kim HJ, Choi HS, Park JH, et al. Factors influencing the technical difficulty of endoscopic clearance of bile duct stones. Gastrointest Endosc 2007;66:1154-1160.

7. Binmoeller KF, Bruckner M, Thonke F, Soehendra N. Treatment of difficult bile duct stones using mechanical, electrohydraulic and extracorporeal shock wave lithotripsy. Endoscopy 1993;25:201206.

8. Keizman D, Shalom MI, Konikoff FM. An angulated common bile duct predisposes to recurrent symptomatic bile duct stones after endoscopic stone extraction. Surg Endosc 2006;20:1594-1599.

9. Kim DI, Kim MH, Lee SK, et al. Risk factors for recurrence of primary bile duct stones after endoscopic biliary sphincterotomy. Gastrointest Endosc 2001;54:42-48.

10. Cheon YK, Lehman GA. Identification of risk factors for stone recurrence after endoscopic treatment of bile duct stones. Eur J Gastroenterol Hepatol 2006;18:461-464.

11. Keizman D, Ish Shalom M, Konikoff FM. Recurrent symptomatic common bile duct stones after endoscopic stone extraction in elderly patients. Gastrointest Endosc 2006;64:60-65. 